\title{
Chilaiditi's sign in a child with nephrotic syndrome
}

\author{
Kanika Kapoor $\cdot$ Abhijeet Saha $\cdot$ N. K. Dubey
}

Received: 21 November 2012/ Accepted: 13 December 2012/Published online: 27 December 2012

(C) Japanese Society of Nephrology 2012

Keywords Chilaiditi's sign · Children .

Nephrotic syndrome

A 10-year-old male with steroid-resistant nephrotic syndrome presented with abdominal pain, vomiting and massive ascites. An X-ray of the abdomen and chest showed air-filled dilated bowel loops in the subdiaphragmatic area with haustral markings (Fig. 1), which is the classic 'Chilaiditi's sign' [1]. Hepatodiaphragmatic interposition of the colon is mostly diagnosed as an incidental finding on an erect chest or abdominal roentgenogram. Sometimes the patient may present with abdominal pain, nausea, vomiting, bloating, anorexia, diaphoresis, constipation, substernal pain, and even cardiac arrhythmias or respiratory distress [2]. When symptomatic, it is known as Chilaiditi's syndrome. Predisposing factors include chronic constipation, shrunken liver, ascites, phrenic nerve injury and excessive aerophagia [3]. Laxity of suspensory ligaments and elevation of hemidiaphragm due to massive ascites were predisposing factors for redundancy of colon in our patient. This condition can be confused with pneumoperitoneum and subphrenic abscess radiologically. Features that point towards the diagnosis of Chilaiditi's sign on radiography are the presence of haustra or valvulae conniventes and the fixation of the position of the radiolucency when the position of the patient is changed. In some cases computed tomography of the abdomen may be required if diagnosis is

K. Kapoor · A. Saha $(\bowtie) \cdot$ N. K. Dubey

Division of Pediatric Nephrology, Department of Pediatrics, Postgraduate Institute of Medical Education and Research associated Dr Ram Manohar Lohia Hospital, Room No 406, PGIMER Building, New Delhi 110001, India

e-mail: drabhijeetsaha@yahoo.com

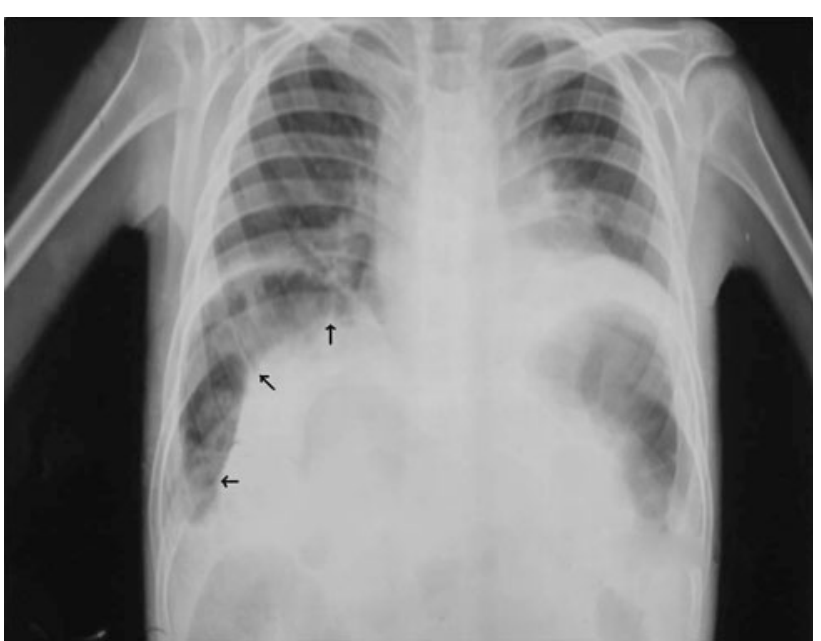

Fig. 1 Erect postero-anterior view of chest X-ray showing right subdiaphragmatic air with haustral markings (arrows)

uncertain. Symptomatic patients usually improve on conservative management; however, colopexy may be required in patients with worsening of symptoms.

Conflict of interest None.

\section{References}

1. Tzimas T, Baxevanos G, Akritidis N. Chilaiditi's sign. Lancet. 2009;373:836.

2. Gulati MS, Wafula J, Aggarwal S. Chilaiditi's sign possibly associated with malposition of chest tube placement. J Postgrad Med. 2008;54:138-9.

3. Joo Young-Eun. Chilaiditi's sign. Korean J Gastroenterol. 2012;59:260-1. 